\title{
Dental License
}

National Cancer Institute

\section{Source}

National Cancer Institute. Dental License. NCI Thesaurus. Code C52649.

The legal right to practice dentistry. 